\title{
Realidad virtual y sus aplicaciones en la enseñanza para personas con discapacidad auditiva
}

\section{Virtual reality and its applications in the teaching of people with hearing disabilities}

\author{
Victor Alfonso Riascos Mendez ${ }^{1}$ (D), Sergio Steban Zuluaga \\ Manrique ${ }^{(D)}$ y Richar Alexander García Mazabuel ${ }^{(D)}$ \\ Grupo de Investigación Sinergia, SENA, Popayán, Colombia
}

Resumen. El Centro de Comercio y Servicios del SENA - Regional Cauca en Colombia, cuenta con una gran demanda de la comunidad con discapacidad auditiva para acceder a la educación media, específicamente en el programa Técnico en Cocina. Por tal motivo, se ha requerido del apoyo de intérpretes en lengua de señas Colombianas como canal de comunicación entre los aprendices con deficiencia auditiva e instructores del área de cocina. Sin embargo, se ha observado en los ambientes de aprendizaje que existe bajo acceso a recursos técnico-pedagógicos a los cuales puedan acudir los aprendices sordos para apoyar sus procesos formativos, y existe la necesidad de herramientas TIC que sirvan como canal de comunicación entre el Español y la lengua de señas colombiana. Por lo anterior, este artículo describe el desarrollo de una aplicación en realidad virtual donde por medio de un avatar interactivo se darán un conjunto de instrucciones en lengua de señas colombianas para preparar recetas, a fin de simular un ambiente de cocina donde se expresará lenguaje técnico el cual deberá ser interpretado tanto por aprendices como instructores. La finalidad de este proyecto es verificar la apropiación efectiva de un conjunto de señas tanto por personas oyentes y no oyentes, a través de una aplicación de realidad virtual.

Palabras Claves. Diseño de videojuegos; educación, gamificación, inteligencia artificial, lenguaje de señas, realidad virtual, videojuegos.

Resumen. The Commerce and Services Center of SENA - Regional Cauca in Colombia, has a high demand from the deaf community to access secondary education, specifically in its Kitchen Technician program. Therefore, the support of interpreters in Colombian Sign Language has been required as a channel of communication between hearing-impaired apprentices and instructors in the kitchen area. However, it has been observed in the learning environments that there is low access to technical-pedagogical resources to which deaf learners can turn to support their training processes, evidencing at the same time the need for ICT tools that serve as a communication channel between Spanish and Colombian Sign Language. Hence, this article describes the development of a virtual reality application in which through an interactive avatar, a set of instructions in Colombian Sign Language will be given to prepare recipes, in order to simulate a kitchen environment where technical language will be expressed to be interpreted by both apprentices and instructors. The purpose of this project is to verify the effective appropriation of a set of signs by both hearing and non-hearing people, through a virtual reality application.

1 victor_riascos@misena.edu.co 
Keywords. Video game Design; education; gamification; artificial intelligence; sign language; virtual reality; video games.

Como Citar. A. V. A. Riascos Mendez, S. S. Zuluaga Manrique, R. A. García Mazabuel, "Realidad virtual y sus aplicaciones en la enseñanza para personas con discapacidad auditiva", Jou. Cie. Ing., vol. 12, no. 1 , pp. 80-93, 2020. doi:10.46571/JCl.2020.1.7

Recibido: 14/10/2019 Revisado: 26/03/2020 Aceptado: 13/04/2020

\section{Introducción}

Según estudios realizados por la Organización Mundial de la Salud (OMS) se estima que el 5\% de la población mundial es caracterizada como población con discapacidad auditiva [1], y según estudios recopilados por el Instituto Nacional para Sordos (INSOR) y el Departamento Nacional de estadística (DANE), aproximadamente el 1\% de la población Colombiana pertenecen a dicha comunidad [2].

Esta comunidad generalmente presenta problemas de aprendizaje dada la baja efectividad en la transmisión de mensajes entre personas oyentes y no oyentes, y en lo concerniente a tecnologías de la información y comunicación, no se cuenta con suficientes materiales educativos computarizados para permitir la comunicación y el aprendizaje autónomo [3-5].

Para mitigar lo anterior, se han desarrollado diversos software informáticos para la población sorda como aplicaciones web [6-8], aplicaciones móviles [9-11], videojuegos [9,12-14], aplicaciones de realidad aumentada [9,15-17], aplicaciones de realidad virtual $[18,19]$, entre otro tipo de soluciones.

Por consiguiente, desde el semillero de investigación Sinergia del Centro de Comercio y Servicios, SENA de la Regional Cauca en Colombia, y como parte de la iniciativa presentada por el Sistema de Investigación, Desarrollo Tecnológico e Investigación (SENNOVA), se desarrolló un videojuego de realidad virtual a fin de mitigar de cierto modo la carencia de materiales educativos computarizados para la comunidad de aprendices sordos que son el público objetivo de este proyecto.

Cocinando enSeñas, es un videojuego de realidad virtual que simula una cocina, en la cual, un avatar interactivo por medio de lengua de señas colombianas le indicará al jugador cómo realizar una serie de pasos para preparar una receta.

\section{Fundamentos teóricos}

\subsection{Educación}

Las TIC juegan un papel decisivo en el proceso de enseñanza-aprendizaje en los diversos entornos educativos $[20,21]$. Sin duda, las nuevas tecnologías pueden suministrar medios para la mejora de los procesos de enseñanza y aprendizaje, y para la gestión de los entornos educativos en general, ya que facilitan la cooperación y colaboración entre alumnos. Además, contribuyen a superar desigualdades sociales [20].

El uso de las TIC, debe integrarse en los procesos de enseñanza convencionales de manera paralela y se deben desarrollar con un enfoque basado en la educación, que prevalezca sobre la innovación de la solución [20,22]. Con el uso de este tipo de entornos educativos computarizados, los estudiantes centran el aprendizaje en sus necesidades, estilos de aprendizaje y preferencias [21].

Debido a que la lengua de señas es el lenguaje natural de la comunidad sorda, y dado que esta lengua se expresa de manera espacial y visual, es necesario generar contenido multimedia para la generación de herramientas educativas computarizadas para la adecuada transmisión de estos mensajes [23]. 


\subsection{Inteligencia artificial}

Los problemas de la inteligencia artificial están divididos en 5 áreas principales: Búsqueda, reconocimiento de patrones, aprendizaje, planeación e inducción [24-26].

Un computador puede hacer en cierto sentido solo lo que le han ordenado hacer. Inclusive, cuando no sabe cómo resolver un problema, se puede programar a una máquina para buscar a través de un largo espacio de soluciones. Con las técnicas de reconocimiento de patrones la eficiencia a menudo puede ser mejorada, al restringir los métodos a problemas adecuados a este tipo de solución [24].

\subsection{Gamificación}

La gamificación se refiere al uso de elementos de diseño de juegos en contextos que usualmente no usan juegos. Se puede hacer uso de la gamificación como una herramienta para mejorar aspectos como la participación y motivación al realizar tareas o actividades [27] [28].

En la gamificación existen distintos tipos de mecánicas, con las cuales se puede realizar un enfoque hacia los objetivos y necesidades de los diferentes actores relacionados a un proceso [28]. Las mecánicas más representativas en gamificación son:

- Autonomía: Permite o facilita la personalización (ejemplo: Control de privacidad, notificaciones, etc.).

- Competencia: Permite la retroalimentación positiva (ejemplo: Progresión, niveles, puntuación, etc.).

- Relación: Facilita la comunicación entre los jugadores (ejemplos: Mensajes, grupos, foros, redes sociales).

La gamificación puede ser usada en ambientes educativos, siendo el primer paso entender el público objetivo y el contexto del problema que se desea resolver. Como siguiente paso se realiza la estructura de la experiencia de usuario, haciendo uso de las diferentes mecánicas que ofrece la gamificación para adaptarlas de la mejor forma para resolver un problema. Después, se analizan los recursos disponibles y la viabilidad de la estructura realizada en pasos anteriores, para finalmente, realizar pruebas con el público objetivo a fin de verificar que el proceso se está realizando de manera adecuada [29].

\subsection{Videojuegos}

Gracias al desarrollo de las tecnologías interactivas, los videojuegos han evolucionado pasando de ser una actividad sinónimo de solo entretenimiento y pasatiempo, para convertirse de manera alternativa en herramientas de apoyo pedagógico, permitiendo el desarrollo de competencias, habilidades y destrezas en los procesos de enseñanza-aprendizaje [30-32].

Varias investigaciones demuestran las ventajas de los videojuegos en cuanto al desarrollo de habilidades cognitivas, sociales/colaborativas, motoras, comunicativas entre otras. Por lo anterior, es importante explorar las posibilidades que los videojuegos introducen en los ambientes educativos y lo beneficios que trae el interactuar de forma dinámica y entretenida con los contenidos $[33,34]$.

Si bien, la mayoría de videojuegos no están orientados a las necesidades únicas de personas con algún tipo de discapacidad, investigaciones demuestran que los videojuegos pueden ser adaptables y tener mayor accesibilidad para estas personas $[35,36]$. En el caso de la presente investigación, se tendrá en cuenta la población con deficiencia auditiva.

Investigaciones validan el impacto positivo en competencias, habilidades y destrezas de las personas sordas, cuando se exploran alternativas como los videojuegos que facilitan el acceso y transmisión de la información, favoreciendo su potencial educativo [37-39]. 


\subsection{Realidad virtual}

Una de las tecnologías que toman mayor impulso e importancia en el ámbito pedagógico actualmente es la realidad virtual [40], que por medio de dispositivos tecnológicos simula un ambiente real de forma tridimensional interactiva en habientes controlados [41], en los cuales el usuario se siente introducido en un ambiente artificial [42].

Estas nuevas herramientas favorecen el acceso a nuevos conocimientos por medio de estímulos multisensoriales (vista, tacto, oídos), creando experiencias que generarán un conocimiento directo, personal, subjetivo e implícito en la medida de lo posible, de manera sencilla, lúdica y formativa, acercando al usuario a casi cualquier materia del conocimiento [43]. Lo anterior es posible ya que la realidad virtual favorece la sensación de inmersión y permite crear experiencias en primera persona.

Aprovechando la capacidad inmersiva de la realidad virtual que se dirige principalmente a los sentidos (vista, oído, tacto) mediante elementos externos (cascos de visualización, controles, sensores de movimiento, etc.), y teniendo en cuenta que el principal canal de comunicación de las personas sordas es el visual, se exploran nuevas posibilidades para generar mecanismos y estrategias de transmisión de información y comunicación que permitan minimizar las barreras existentes entre personas oyentes y no oyentes por medio de las tecnologías, favoreciendo de este modo el proceso de enseñanza-aprendizaje e inclusión social [37, 42,43].

\section{Videojuego de realidad virtual: Cocinando enSeñas}

El presente apartado se centra en describir el proceso de desarrollo del videojuego de realidad virtual, Cocinando enSeñas.

\subsection{Conceptualización}

Para realizar el concepto de alto nivel del videojuego, se conformó un grupo focal compuesto por aprendices sordos egresados del programa Técnico de Cocina del Centro de Comercio y Servicios en Popayán, Colombia, personas con deficiencia auditiva interesadas en el proyecto, interpretes en lengua de señas colombianas, instructores del programa y el equipo de desarrollo del proyecto.

El primer paso fue realizar un proceso de gamificación donde se validaron de manera previa las mecánicas básicas de juego. En este proceso se tuvo en cuenta para crear un intermediario con el intérprete de lengua de señas, así como un canal de comunicación entre el equipo de desarrollo y los usuarios finales del proyecto.

Al finalizar el proceso de gamificación con la comunidad sorda interesada en el proyecto, se pudo determinar cuál sería la interacción con los elementos del mundo, cómo se comunicarían con el intérprete, los objetivos de juego, las recompensas, entre otros aspectos intrínsecos del juego.

\subsection{Diseño}

A partir de la recolección de información al realizar la gamificación, se documentó de manera estructurada y formal el videojuego por medio de un documento de diseño [44], definiendo las características a implementar, tales como: género, público objetivo, estilo visual, alcance del proyecto, historia y narrativa, diseño y definición de personajes, mecánicas de juego, interfaces, aspectos técnicos, entre otros [45].

El concepto de alto nivel, está relacionado a desarrollar un videojuego en realidad virtual donde el jugador tomará el papel de un cocinero bajo las órdenes de un avatar interactivo que se comunicará por medio de lenguaje de señas.

El diseño del personaje principal (Figura 1) se basó en las sugerencias de la comunidad sorda, por tal motivo, se definió al avatar interactivo como un roedor mágico, esto con la intención de generar empatía con los jugadores. Además su estructura se asemeja a la de un humano al tener la capacidad de realizar movimientos corporales y faciales fácilmente reconocibles. 
Otro aspecto que se tuvo en cuenta al diseñar el personaje fue la selección de la gama de colores. Constantemente los usuarios finales del sistema otorgaron retroalimentación para que existiera contraste entre las manos y el cuerpo.

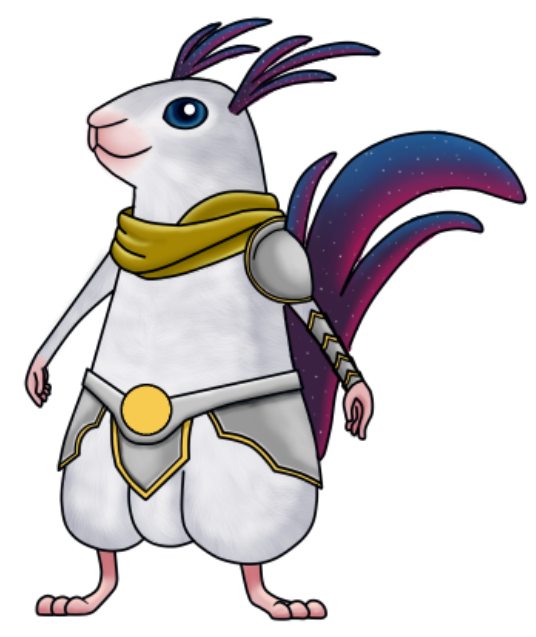

Figura 1: Concepto final del intérprete interactivo, según las apreciaciones brindadas por parte de la comunidad sorda adscrita al proyecto.

\subsection{Desarrollo de la aplicación}

Para el proceso de desarrollo de la aplicación se realizaron dos actividades en paralelo, el desarrollo de los recursos gráficos $(2 \mathrm{D} / 3 \mathrm{D})$ y el desarrollo software (aplicación de realidad virtual).

3.3.1. Desarrollo de recursos gráficos. Para el desarrollo de los recursos gráficos se tuvo en cuenta el diseño del personaje y los elementos plasmados en el documento de diseño de juego para generar una estética agradable y uniforme entre sí.

Además, se indagó acerca de procesos de topología, texturizado, rigging, skinning, controladores, shape keys, drives, animación y compatibilidad al integrar en el motor de desarrollo de videojuegos seleccionado. Este estudio previo permitió establecer una correcta topología tridimensional y una adecuada distribución de la estructura de huesos para realizar las animaciones por parte del personaje.

Posterior a ello, se realizó el blueprint (Figura 2) como representación bidimensional del personaje siendo observado de manera frontal y lateral, utilizando este recurso como referencia para la generación del modelo tridimensional [46,47].

A partir del blueprint, se realizó un modelo tridimensional base con figuras geométricas, las cuales fueron deformadas para generar la estructura base. La estructura base se combinó con el fin de generar una geometría, con la cual se definió un modelo tridimensional en alto poligonaje a través de la realización de un proceso de escultura digital, que permitió definir de manera detallada al personaje.

Para obtener un modelo tanto apto para animación como óptimo para el motor de desarrollo de videojuegos, se realizó un proceso de retopología para generar un modelo tridimensional de bajo poligonaje (Figura 3 ). 


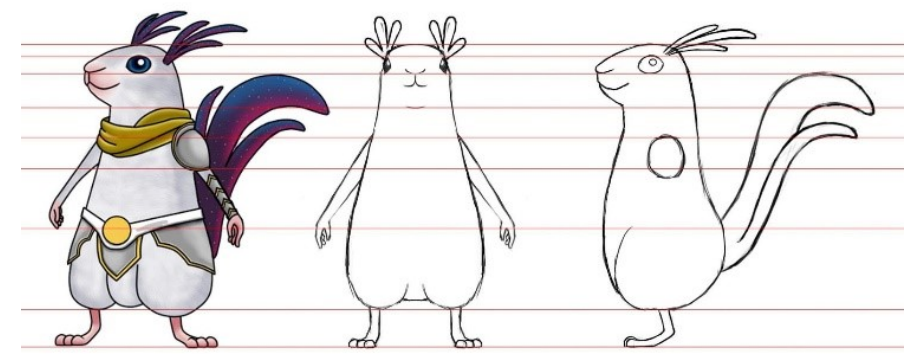

Figura 2: Blueprint del personaje principal.

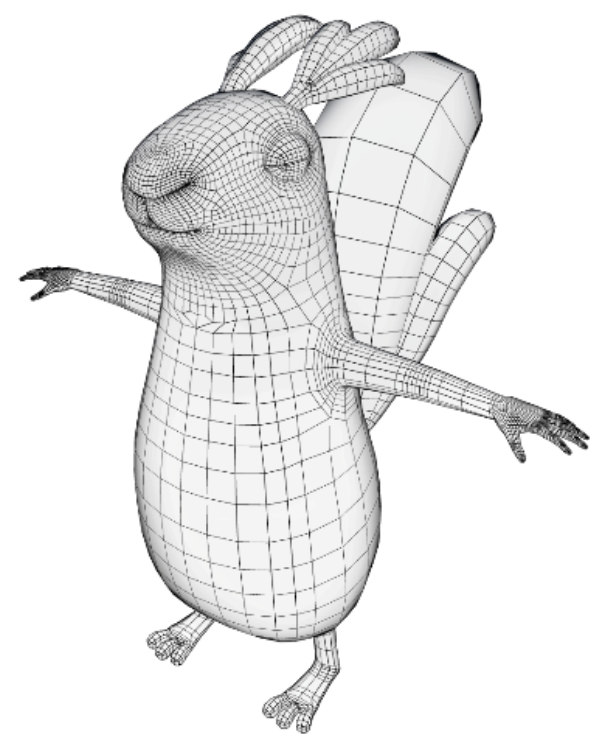

Figura 3: Malla en bajo poligonaje del personaje principal.

Cabe destacar, que el modelo en bajo poligonaje contiene mayor cantidad de polígonos tanto en las manos como en la cara, debido que dichas partes tendrán movimientos más complejos que el resto del cuerpo.

Una vez construido el modelo de baja resolución, se genera el mapa UV (Figura 4), que es la proyección del modelo tridimensional a un plano bidimensional.

Para realizar las animaciones del personaje, fue necesario realizar una estructura de huesos (rigging) (Figura 5) y a cada elemento de la estructura, asignarle la influencia a un conjunto de polígonos (skinning).

El resultado del flujo de trabajo expuesto en el presente apartado es el observado en la figura 6 del presente documento.

Finalmente, se determinó un conjunto de acciones que realizará el avatar interactivo para indicar paso a paso la preparación de una receta de cocina, dando paso al proceso de animación del personaje por parte del equipo de desarrollo.

Cada una de las animaciones realizadas pasó por un proceso de validación con la comunidad sorda del centro de formación con el fin de evitar ambigüedad en la entrega de la información por parte del avatar interactivo (Figura 7). 


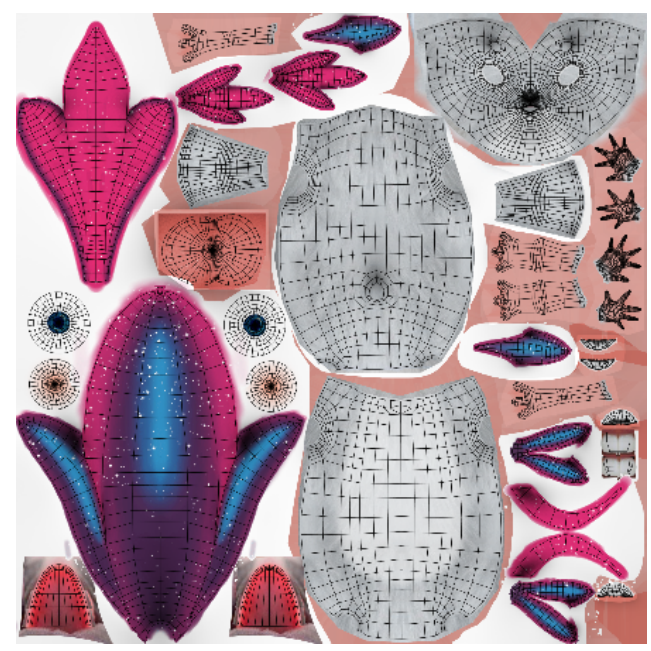

Figura 4: Mapa UV del personaje principal del videojuego.

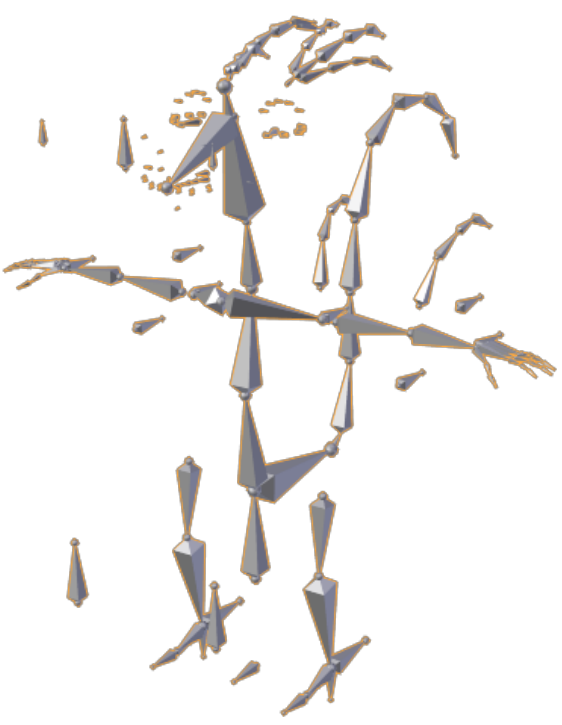

Figura 5: Estructura de huesos que conforman al personaje tridimensional.

3.3.2. Desarrollo del prototipo Para el desarrollo del proyecto Cocinando enSeñas, se hizo uso del proceso de desarrollo software Scrum [48]. El equipo de desarrollo desglosó el documento de diseño de juego para generar las historias de usuario a desarrollar como parte del product backlog. Cabe destacar que mientras se avanzaba en el producto, nuevas historias de usuario surgieron o se tuvieron que complementar.

Seguidamente, el equipo de desarrollo estableció reuniones con los usuarios finales para determinar las historias de usuario más relevantes a desarrollar en cada release backlog. En el release backlog se priorizó y se estimó el tiempo en horas de desarrollo para cada historia de usuario.

Nuevamente, las historias de usuario asignadas al release backlog se dividieron en un conjunto de hitos de corta duración, denominados sprint backlogs. Al realizar cada historia de usuario 


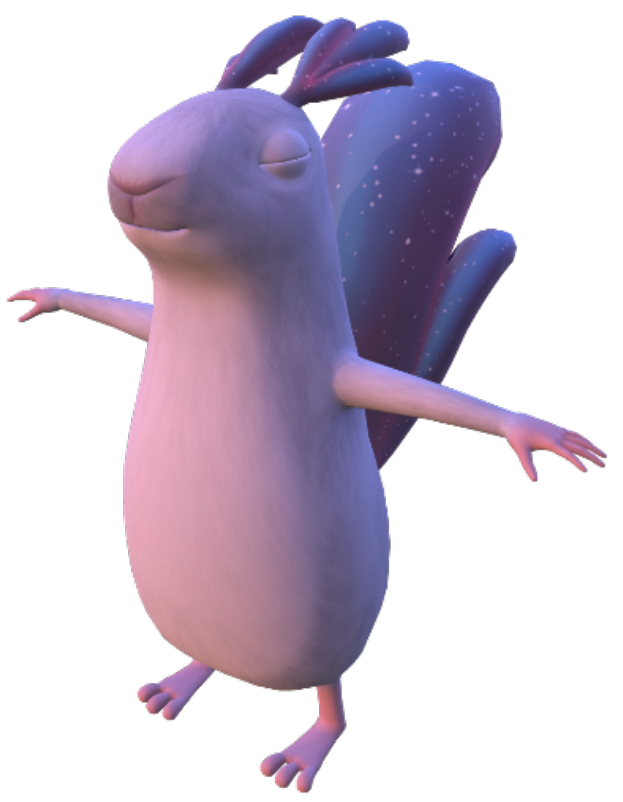

Figura 6: Avatar interactivo que ejecutará animaciones en lengua de señas colombianas.

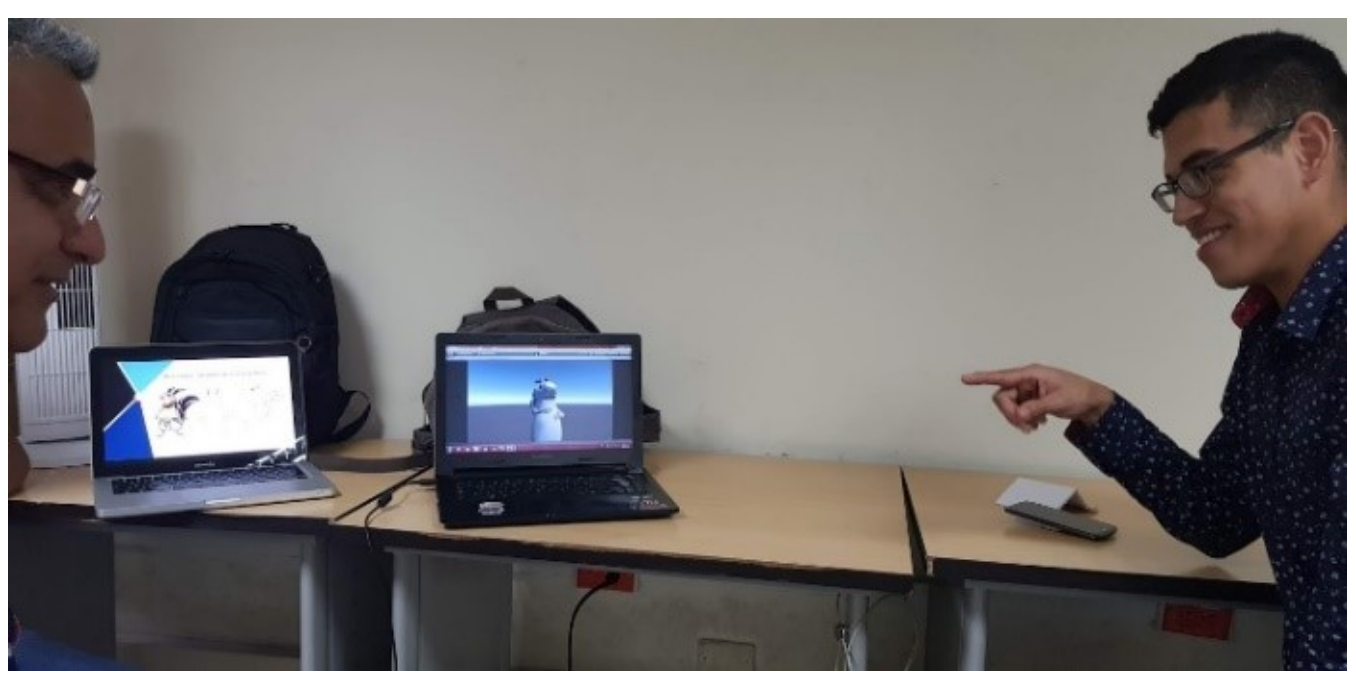

Figura 7: Miembro de la comunidad sorda dando retroalimentación sobre las animaciones desarrolladas.

del sprint se obtuvieron productos gráficos y computacionales listos para ser probados.

Adicionalmente, el equipo de desarrollo realizó reuniones diarias donde cada uno de los miembros comentaba los avances en las historias de usuario, los inconvenientes presentados y las soluciones a los problemas al realizar las actividades.

Algunos de los release backlogs más relevantes para obtener un videojuego funcional fueron:

- Módulo de realidad virtual: Este módulo se centró en integrar los recursos tridimensionales al motor de desarrollo de videojuegos. Cada uno de los recursos gráficos tuvo que ser generado según escalas reales, para así generar mayor inmersión en el jugador y percepción de estar en una cocina real (Figura 8). 


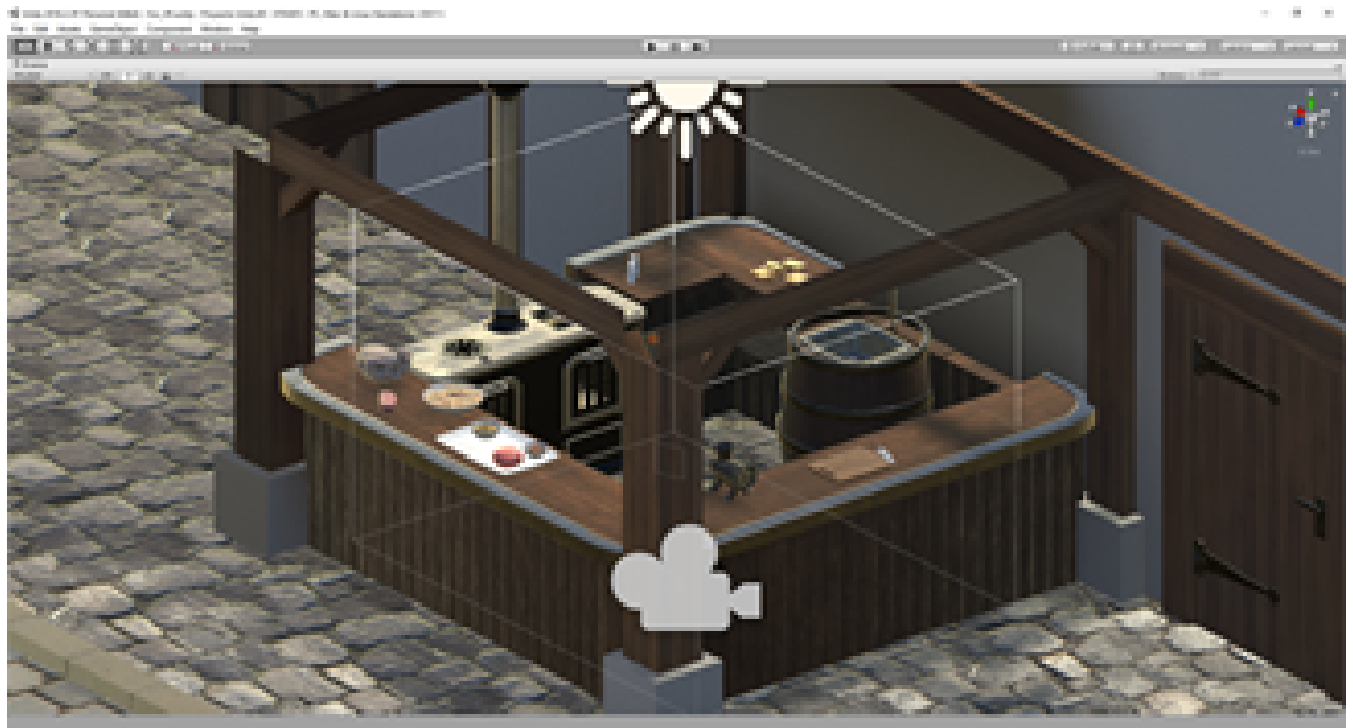

Figura 8: Escena de juego con los recursos gráficos integrados.

El proyecto fue desarrollado para el dispositivo HTC Vive, por lo cual, la lógica de programación se enfocó a obtener las entradas de usuario por medio de los controles que provee dicho dispositivo (Figura 9).

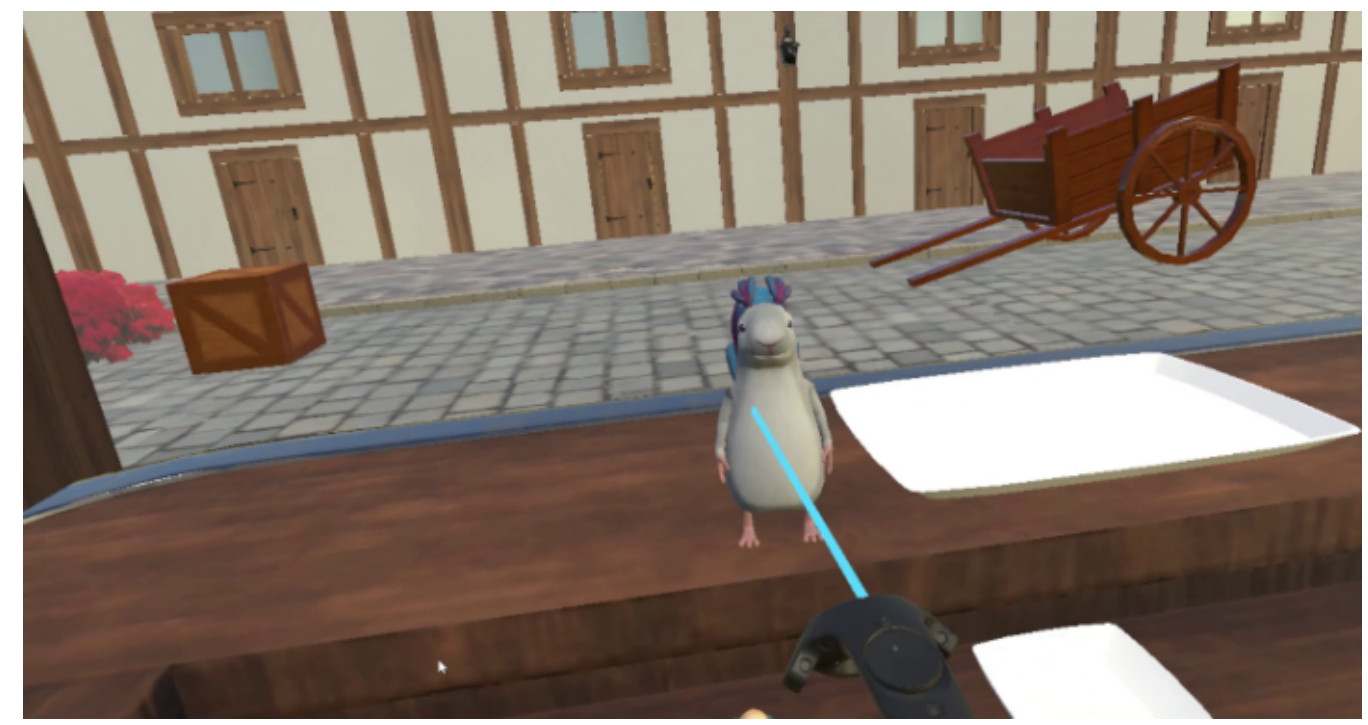

Figura 9: Videojuego de realidad virtual Cocinando enSeñas.

- Máquina de estados finitos: Para verificar el estado en el cual se encontraba la ejecución de acciones para realizar una receta, se hizo uso de una máquina de estados finitos. Está máquina de estados se retroalimentó de las entradas de usuario: si el paso a seguir coincidía con el realizado por el jugador entonces la máquina de estados pasaba al siguiente estado, enviándole un mensaje al avatar interactivo para determinar su acción futura. En caso contrario, la máquina de estados determinaba una manera de retroalimentar al usuario por medio del avatar interactivo. 
- Inteligencia artificial del personaje principal: El avatar interactivo contiene una inteligencia artificial que reacciona al número de interacciones que realiza el usuario para ejecutar una acción de manera correcta. De esta manera, el avatar interactivo al verificar el modelo de aprendizaje del usuario responde de forma adecuada en lengua de señas colombiana.

En el caso de que el usuario requiera de múltiples interacciones para interpretar una seña, la inteligencia artificial del avatar interactivo comprenderá que el usuario no ha comprendido el mensaje, por lo cual, dará retroalimentación visual por medio de una animación bidimensional donde se observará la acción a realizar por parte del usuario.

En el caso que el usuario no capte el mensaje realizado por el avatar de manera visual, se dará paso a especificar el mensaje de manera textual (Figura 10).

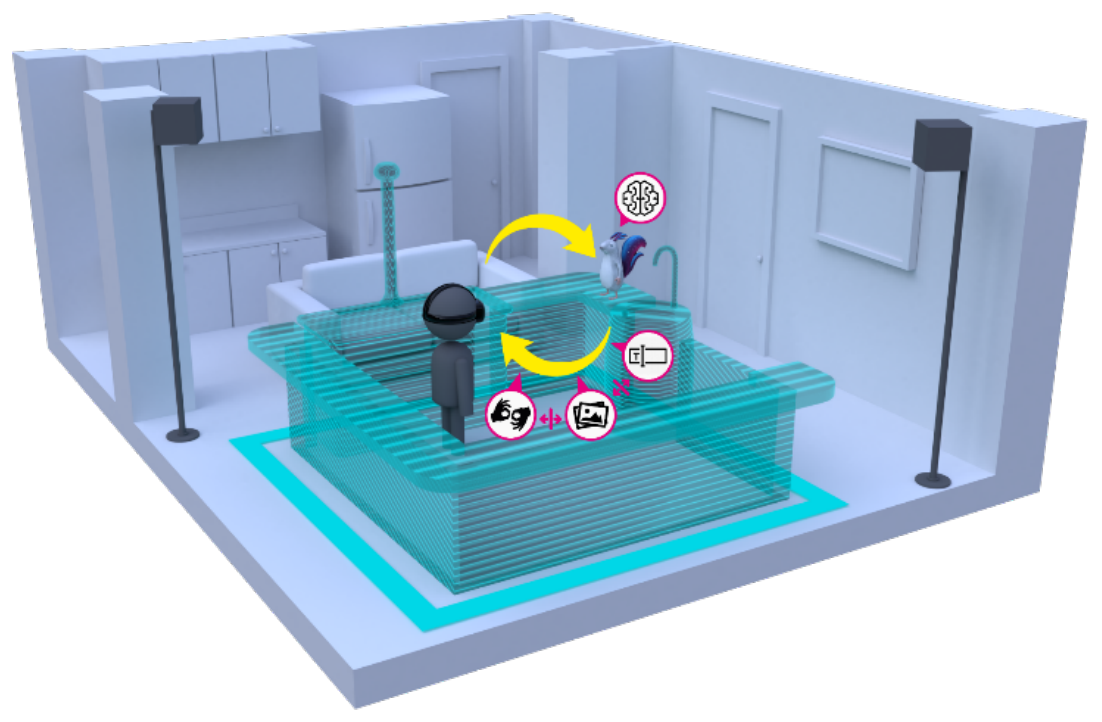

Figura 10: Ciclo de retroalimentación dentro del entorno virtual.

Cabe destacar, que el tipo de retroalimentación por parte de la inteligencia artificial tendrá repercusión en la puntuación del usuario al final de la partida. Con base a dicha puntuación, se determinará el nivel de captación de la información recibida por parte del avatar interactivo.

\section{Pruebas}

Para realizar la validación del proyecto, se tuvieron en cuenta los aprendices sordos del programa Técnico en Cocina del Centro de Comercio y Servicios, SENA - Regional Cauca, y personas sordas pertenecientes a áreas afines e interesados en el proyecto.

La prueba involucró a ocho personas con edades cronológicas entre los 15 y 44 años, con una media de edad de 23 años. Esta práctica contó con un periodo de cuatro horas, en la cual se realizó una encuesta para medir los aprendizajes previos y caracterizar a las personas involucradas en la prueba. Posterior a esto, se hizo la interacción con la aplicación Cocinando enSeñas con las personas sordas y se finalizó la actividad con una encuesta para verificar resultados una vez se interactuó con la aplicación.

4.1. Aprendizajes previos y caracterización de la población sorda involucrada en la prueba Según los datos recolectados en la encuesta, el $25 \%$ de las familias de las personas encuestadas se comunican por medio del español, por lo cual no hacen uso de lengua de señas a pesar de contar 
con un miembro de la familia que requiere ese tipo de comunicación. El $75 \%$ de las familias, mezclan español y lengua de señas para comunicarse e interactuar con el familiar miembro de la comunidad sorda.

Por otro lado, todas las personas sordas encuestadas hacen uso de lengua de señas colombianas, pero debido a que las personas oyentes no dominan dicho lenguaje, el $37.5 \%$ de los encuestados vocalizan o gesticulan sonidos para intentar comunicarse de manera correcta. El $25 \%$ hace uso de apoyo escrito mediante papel y ese mismo porcentaje requiere como intermediarios intérpretes en lengua de señas colombiana. El 12.5\% se apoya en medios digitales escritos para comunicarse y el resto porcentual trata de buscar diversas alternativas para comunicarse de manera asertiva con las personas oyentes.

Otro aspecto a tener en cuenta es que el $75 \%$ de los encuestados manifiestan que no existen suficientes medios de accesibilidad para la comunicación entre personas oyentes y no oyentes, de los cuales el $62.5 \%$ manifiesta que conocen por lo menos una aplicación accesible para este tipo de comunidad. Sin embargo, el $50 \%$ considera que las aplicaciones no cumplen con las expectativas de comunicación e interacción para este tipo de comunidad.

Finalmente, se determinó que los materiales educativos comúnmente utilizados para aprender una nueva área de conocimiento son los videos y los libros. El 87.5\% de los encuestados prefieren observar videos y el $12.5 \%$ hacen uso de libros. Cabe destacar, que muchos de los encuestados han tenido acceso al sistema educativo colombiano, por lo cual han aprendido las dinámicas educativas que requieren para su proceso formativo.

\subsection{Resultados al interactuar con la aplicación de realidad virtual.}

Una vez se realizaron las pruebas con la aplicación, se realizó una encuesta que arrojó los siguientes resultados:

- A pesar que las personas encuestadas tienen un promedio de edad de 23 años, el $62.5 \%$ manifiesta que no hace uso de videojuegos en su diario vivir.

- El 50\% nunca había hecho uso de dispositivos de realidad virtual antes de realizar las pruebas de usuario del proyecto Cocinando enSeñas.

- El 63\% de las personas participantes en las pruebas de usuario coincidieron que la aplicación no fue fácil de usar debido que no tenían experiencias previas con los dispositivos de realidad virtual. Sin embargo, una vez adaptados al modo de interacción pudieron cumplir con los objetivos planteados en el videojuego.

- La totalidad de los encuestados expresó su satisfacción al usar la aplicación Cocinando enSeñas y comentaron que el lenguaje facial y corporal realizado por el avatar interactivo transmitía las señas de manera clara y concisa.

\section{Conclusiones}

Para la adecuada ejecución de este proyecto fue necesario contar con un intérprete de lengua de señas colombiana y miembros de la comunidad con deficiencia auditiva, tanto para definir las animaciones como para corregirlas de manera constante, evitando de este modo ambigüedades en la entrega y recepción de la información.

Por lo anterior, la validación de manera constante con el público objetivo permitió obtener retroalimentación en etapas tempranas de desarrollo y generó así un producto según las necesidades de los usuarios finales del sistema.

Según las pruebas de la aplicación, es necesario contar con un tutorial de juego que explique las mecánicas, reglas de juego y modos de interacción, evitando así, la intervención de un intérprete en lengua de señas en esta actividad. 
Debido la satisfacción de los usuarios con la aplicación, se concluye que el desarrollo de materiales educativos computarizados para población con discapacidad auditiva podría apoyar de manera positiva los procesos formativos en distintas áreas de conocimiento.

\section{Agradecimientos}

Agradecemos al Centro de Comercio y Servicios del SENA regional Cauca y al Sistema de Investigación, Desarrollo Tecnológico e Innovación (SENNOVA) por el apoyo recibido en la ejecución del proyecto.

Agradecemos a Alberto Arango Parra por sus recomendaciones y aporte al componente técnico en cocina, y a Harol Ned Benavides como intérprete en lengua de señas colombianas, que nos permitió comunicarnos con el público objetivo del proyecto. También agradecemos al instructor Jonatan Lemos por sus comentarios y edición del presente artículo.

Y en especial, agradecemos a los aprendices de la comunidad sorda, por retroalimentar el proyecto y realizar las pruebas de usuario, para que así, este proyecto pueda ser usado en beneficio de su comunidad. Sin su valiosa ayuda y apoyo, este proyecto no hubiera sido posible.

\section{Referencias}

[1] "Deafness and hearing loss." Available: http://www.who.int/news-room/fact-sheets/detail/deafness-andhearing-loss. World Health Organization, 15 March 2018 [Online].

[2] "Estadísticas básicas población sorda colombiana - observatorio social." Available: http://www.insor.gov.co/observatorio/estadisticas-basicas-poblacion-sorda-colombiana/. Accessed: 20-Nov-2018, [Online].

[3] C. Guimarães, D. R. Antunes, L. S. García, L. M. Peres, and S. Fernandes, "Pedagogical architecture-internet artifacts for bilingualism of the deaf (sign language/portuguese)," in 2013 46th Hawaii International Conference on System Sciences, pp. 40-49, IEEE, 2013.

[4] H. Salehi and Z. Salehi, "Integration of ict in language teaching: Challenges and barriers," in Proceedings of the 3rd International Conference on e-Education, e-Business, e-Management and e-Learning (IC4E, 2012), IPEDR, vol. 27, pp. 215-219, 2012.

[5] C. Lucas, The sociolinguistics of the deaf community. Elsevier, 2014

[6] M. Jemni, O. Elghoul, and S. Makhlouf, "A web-based tool to create online courses for deaf pupils," in International conference on interactive mobile and computer aided learning, Amman, Jordan, pp. 18-20, 2007.

[7] M. Debevc, P. Kosec, and A. Holzinger, "Improving multimodal web accessibility for deaf people: sign language interpreter module," Multimedia Tools and Applications, vol. 54, no. 1, pp. 181-199, 2011.

[8] A. R. Costa, G. P. Dimuro, et al., "Supporting deaf sign languages in written form on the web," in 10th World Wide Web Conference, Hong Kong, Home Page of Web and Society Track, http://www10. org/cdrom/posters/frame. html. Processing", Written Form on the Web, 2001.

[9] A. P. G. Valencia, "El chef enseña, 1st ed," 2018.

[10] . S. Carolina, "Diseño de la aplicación de lengua de seÑas colombiana para dispositivos mÓviles," Application Design Sign Language Colombian for Mobile Devices.

[11] S. M. Halawani, "Arabic sign language translation system on mobile devices," IJCSNS International Journal of Computer Science and Network Security, vol. 8, no. 1, pp. 251-256, 2008.

[12] A. Melonio and R. Gennari, "How to design games for deaf children: Evidence-based guidelines," in 2nd International Workshop on Evidence-based Technology Enhanced Learning, pp. 83-92, Springer, 2013.

[13] D. Buckley, C. Codina, P. Bhardwaj, and O. Pascalis, "Action video game players and deaf observers have larger goldmann visual fields," Vision research, vol. 50, no. 5, pp. 548-556, 2010.

[14] F. Soltani, F. Eskandari, and S. Golestan, "Developing a gesture-based game for deaf/mute people using microsoft kinect," in 2012 Sixth International Conference on Complex, Intelligent, and Software Intensive Systems, pp. 491-495, IEEE, 2012.

[15] N. M. M. Zainuddin, H. B. Zaman, and A. Ahmad, "Developing augmented reality book for deaf in science: the determining factors," in 2010 International Symposium on Information Technology, vol. 1, pp. 1-4, IEEE, 2010.

[16] N. M. M. Zainuddin, H. B. Zaman, and A. Ahmad, "Heuristic evaluation on augmented reality courseware for the deaf," in 2011 International Conference on User Science and Engineering (i-USEr), pp. 183-188, IEEE, 2011. 
[17] M. R. Mirzaei, S. Ghorshi, and M. Mortazavi, "Combining augmented reality and speech technologies to help deaf and hard of hearing people," in 2012 14th Symposium on Virtual and Augmented Reality, pp. 174-181, IEEE, 2012.

[18] D. Passig and S. Eden, "Enhancing the induction skill of deaf and hard-of-hearing children with virtual reality technology," Journal of Deaf Studies and Deaf Education, vol. 5, no. 3, pp. 277-285, 2000.

[19] J. R. F. Brega, I. A. Rodello, D. R. C. Dias, V. F. Martins, and M. de Paiva Guimarães, "A virtual reality environment to support chat rooms for hearing impaired and to teach brazilian sign language (libras)," in 2014 IEEE/ACS 11th International Conference on Computer Systems and Applications (AICCSA), pp. 433-440, Ieee, 2014.

[20] S. L. A. A. de Innovacion y Desarrollo, "3c tic: cuadernos de desarrollo aplicados a las tic.," 3ciencias, vol. 2 , no. $1,2013$.

[21] P. R. Humanante-Ramos, F. J. García-Peñalvo, and M. Á. Conde-González, "Electronic devices and web 2.0 tools: usage trends in engineering students," International Journal of Engineering Education (IJEE), vol. 33, no. 2B, pp. 790-796, 2017.

[22] L. R. Malbernat, "Tics en educación: competencias docentes para la innovación en pos de un nuevo estudiante," in VI Congreso de Tecnología en Educación y Educación en Tecnología, 2011.

[23] M. Jemni and O. Elghoul, "Using ict to teach sign language," in 2008 Eighth IEEE International Conference on Advanced Learning Technologies, pp. 995-996, IEEE, 2008.

[24] M. Minsky, "Steps toward artificial intelligence," Proceedings of the IRE, vol. 49, no. 1, pp. 8-30, 1961.

[25] N. J. Nilsson, "Artificial intelligence stuart russell and peter norvig, artijcial intelligence: A modem approach*," vol. 3702, no. 96, 1996.

[26] S. T. S. T. P. Pridmore, S. R. Pollard and M. V. Someren, "Artificial intelligence techniques," pp. 21-22, 1997.

[27] S. Deterding, M. Sicart, L. Nacke, K. O'Hara, and D. Dixon, "Gamification. using game-design elements in non-gaming contexts," in CHI'11 extended abstracts on human factors in computing systems, pp. 2425$2428,2011$.

[28] A. F. Aparicio, F. L. G. Vela, J. L. G. Sánchez, and J. L. I. Montes, "Analysis and application of gamification," in Proceedings of the 13th International Conference on Interacción Persona-Ordenador, pp. 1-2, 2012.

[29] W. Hsin-Yuan Huang and D. Soman, "A practitioner's guide to gamification of education," Rotman School of Management, University of Toronto, 2013.

[30] J. Parong, R. E. Mayer, L. Fiorella, A. MacNamara, B. D. Homer, and J. L. Plass, "Learning executive function skills by playing focused video games," Contemporary Educational Psychology, vol. 51, pp. 141$151,2017$.

[31] H. J. Brown, Videogames and education. ME Sharpe, 2008.

[32] K. Durkin, J. Boyle, S. Hunter, and G. Conti-Ramsden, "Video games for children and adolescents with special educational needs," Zeitschrift für Psychologie, 2015.

[33] B. Manero, J. Torrente, Á. Serrano, I. Martínez-Ortiz, and B. Fernández-Manjón, "Can educational video games increase high school students' interest in theatre?," Computers $\&$ education, vol. 87, pp. 182-191, 2015 .

[34] I. Granic, A. Lobel, and R. C. Engels, "The benefits of playing video games.," American psychologist, vol. 69, no. 1, p. $66,2014$.

[35] R. Ocker, "Achieving immersive gameplay: Interpreters and video game inaccessibility," 2017.

[36] Y. Bouzid, M. A. Khenissi, and M. Jemni, "Designing a game generator as an educational technology for the deaf learners," in 2015 5th International Conference on Information $\&$ Communication Technology and Accessibility (ICTA), pp. 1-6, IEEE, 2015.

[37] H. Nagendra, V. Kumar, and S. Mukherjee, "Evaluation of cognitive behavior among deaf subjects with video game as intervention," Cognitive Systems Research, vol. 42, pp. 42-57, 2017.

[38] K. F. Li, K. Lothrop, E. Gill, and S. Lau, "A web-based sign language translator using 3d video processing," in 2011 14th International Conference on Network-Based Information Systems, pp. 356-361, IEEE, 2011.

[39] N. T. CICANT, "The centre for research in applied communication," Culture, Proceedings of Play2Learn 2018, 2018.

[40] E. Gandolfi, Virtual Reality and Augmented Reality, pp. 545-561. 042018.

[41] M. Jedd, "Virtual reality," PM Netw, vol. 20, no. 8, pp. 58-63, 2006.

[42] C. Christou, Virtual reality in education, pp. 228-243. IGI Global, 2010.

[43] I. D. Curcio, A. Dipace, and A. Norlund, "Virtual realities and education," Research on Education and Media, vol. 8, no. 2, pp. 60-68, 2016.

[44] J. Schell, The Art of Game Design: A book of lenses. CRC press, 2008.

[45] S. Rogers, Level Up! The guide to great video game design. John Wiley \& Sons, 2014.

[46] J. Williamson, Character development in Blender 2.6. Course Technology PTR, 2010. 
[47] C. Totten, Game Character Creation with Blender and Unity. John Wiley \& Sons, 2012.

[48] "Homepage - scrum.org." Available: https://www.scrum.org/. Accessed: 19-Nov-2018, [Online].

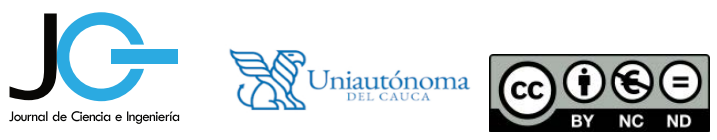

